\title{
Nile grass rats see the light of day
}

\author{
Peter Flanagan, DVM
}

\section{SCIENTIFIC NAME \\ Arvicanthis niloticus}

\section{TAXONOMY}

PHYLUM: Chordata

CLASS: Mammalia

ORDER: Rodentia

FAMILY: Muridae

\section{Physical description}

As its common name suggests, the Nile grass rat is native to the grasslands of sub-Saharan Africa and the southern Arabian Peninsula ${ }^{1}$. This small, stocky rodent has a generally rounded appearance. Females are slightly smaller than males, with average weights of $115 \mathrm{~g}$ and $120 \mathrm{~g}$, respectively. The approximate body length is $11-20 \mathrm{~cm}$ with the tail adding another $9-15 \mathrm{~cm}$ to the animal's overall length. Nile grass rats usually live for about 2 years in captivity.

The Nile grass rat body is covered by a coat of brown-gray fur incorporating tricolor hairs that are brownish at the base, paler in the center and tipped with black. The pronounced agouti coloring of the coarse, stiff hairs gives the coat a rough, almost spiny appearance. Many Nile grass rats have a distinct dark dorsal stripe running along the spine and the top of the tail. The tail is typically covered by very fine, short, stiff hairs, which is unusual for rodents.

\section{Reproduction and husbandry}

Most research facilities can accommodate Nile grass rats easily because they are small and reproductively efficient. Like many rodent species, female grass rats are polyestrous, spontaneous ovulators that are capable of breeding year-round under favorable conditions. Females usually bear four to six pups after a gestation period of 21-24 days. At birth, the fully furred Nile grass rats are more precocious than the common laboratory rodents.

\section{Research résumé}

Nile grass rats differ from most common laboratory rodents in behavior and physiology. Most notably, Nile grass rats are diurnal, leading some researchers to propose that this species may be a more appropriate animal model for other diurnal species, particularly humans ${ }^{2}$. Some initial studies focused on identifying the neurological signals that contribute to differences in behavioral and physiological patterns between diurnal and nocturnal mammals ${ }^{3}$. Subsequent studies have examined how social interactions within and between

Flanagan is Senior Clinical Veterinarian in the Department of Animal Medicine, University of Massachusetts Medical School, Worcester, MA.

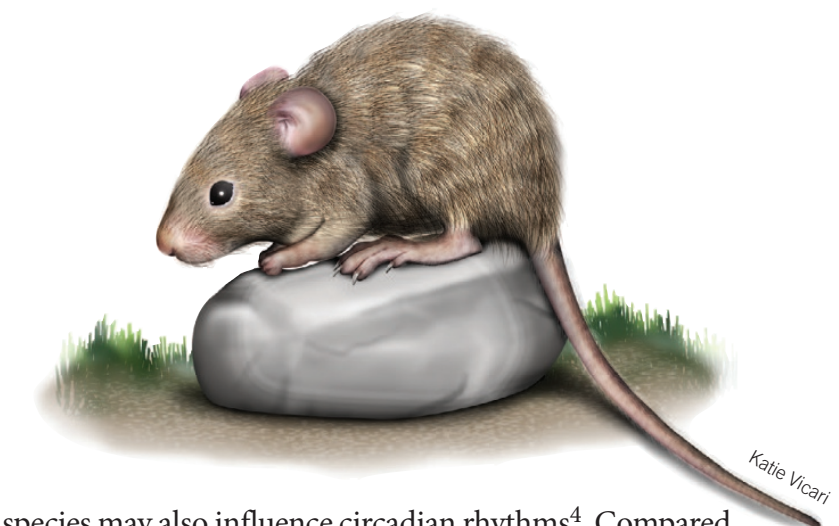

species may also influence circadian rhythms ${ }^{4}$. Compared with nocturnal rodents, Nile grass rats have a higher concentration of cone photoreceptors in the retina ${ }^{5}$, making this species an appropriate model for retinal diseases in humans.

Nile grass rats are increasingly used to study the effects of external stressors, such as diet, on the development of certain metabolic conditions. These studies are based on the observation that Nile grass rats fed a conventional rodent diet often spontaneously develop type 2 diabetes. Most animals develop hyperglycemia by one year of age, although certain subpopulations show elevated blood glucose levels earlier, depending on growth rate, gender and $\operatorname{diet}^{6}$. Related studies indicate that Nile grass rats also develop conditions such as obesity, dyslipidemia, hyperinsulinemia and hypertension ${ }^{7}$. Each of these clinical signs falls within the constellation of metabolic syndrome, which is increasingly recognized in humans consuming the western diet. Although genetically engineered rodents are available for studying metabolic syndrome, none recapitulate all the components of this complex disease. The Nile grass rat may be the most appropriate animal model for studying the complex interactions between genes and diet that are thought to produce this multifaceted condition.

1. Nowak, R. Walker's Mammals of the World 6th edn. 1603-1604 (The Johns Hopkins University Press, Baltimore and London, 1999).

2. Refinetti, R. The Nile grass rat as a laboratory animal. Lab Anim. (NY) 33, 54-57 (2004).

3. Schwartz, M.D., Nunez, A.A. \& Smale, L. Rhythmic cFos expression in the ventral subparaventricular zone influences general activity rhythms in the Nile grass rat, Arvicanthis niloticus. Chronobio. Int. 7, 1290-1306 (2009).

4. Castillo-Ruiz, A., Paul, M.J. \& Schwartz, W.J. In search of a temporal niche: social interactions. Prog. Brain Res. 199, 267-280 (2012).

5. Gaillard, F., Kuny, S. \& Sauve, Y. Topographic arrangement of S-cone photoreceptors in the retina of the diurnal Nile grass rat (Arvicanthis niloticus). Invest. Ophthalmol. Vis. Sci. 50, 5426-5434 (2009).

6. Chaabo, F., Pronczuk, A., Mastova, E. \& Hayes, K.C. Nutritional correlates and dynamics of diabetes in the Nile rat (Arvicanthis niloticus): a novel model for diet-induced type 2 diabetes and the metabolic syndrome. Nutr. Metab. 7, 29-40 (2012).

7. Noda, K. et al. An animal model of spontaneous metabolic syndrome: Nile grass rat. FASEB J. 24, 2443-2453 (2012). 